\title{
Measurements of the asymmetric, dynamic sheath around a pulse biased sphere immersed in flowing metal plasma
}

\author{
Hongchen $\mathrm{Wu}^{1,2,3}$ and André Anders ${ }^{3}$ \\ ${ }^{1}$ Physics Department, Dalian University of Technology, Dalian 116024, P. R. China \\ ${ }^{2}$ Beijing Aeronautical Manufacturing Technology Research Institute, P.O. Box 863 Beijing \\ 100024, P. R. China \\ ${ }^{3}$ Lawrence Berkeley National Laboratory, University of California, 1 Cyclotron Road, \\ Berkeley, California 94720, USA
}

\begin{abstract}
.
A long-probe technique was utilized to record the expansion and retreat of the dynamic sheath around a spherical substrate immersed in pulsed cathode arc metal plasma. Positively biased, long cylindrical probes were placed on the side and downstream of a negatively pulsed biased stainless steel sphere of 1" $(25.4 \mathrm{~mm})$ diameter. The amplitude and width of the negative high voltage pulses (HVP) were $2 \mathrm{kV}, 5 \mathrm{kV}, 10 \mathrm{kV}$, and $2 \mu \mathrm{s}, 4 \mu \mathrm{s}, 10 \mu \mathrm{s}$, respectively. The variation of the probe (electron) current during the HVP is a direct measure for the sheath expansion and retreat. Maximum sheath sizes were determined for the different parameters of the HVP. The expected rarefaction zone behind the biased sphere (wake) due to the fast plasma flow was clearly established and quantified.
\end{abstract}

Key words: plasma probe, sheath measurement, cathodic arc plasma, wake effect 


\section{Introduction}

Metal (and carbon) plasmas have their special place in many industrial processes such as the deposition of ultrathin layers in the computer and data storage industry [1-3], the coating and filling of sub-micron trenches [4-6], and the synthesis of nanocomposite materials [7-10]. The cathodic vacuum arc discharge is a convenient and well-known method to produce flowing metal plasma. Such plasma has the advantage that it is practically fully ionized and therefore biasing of a substrate is very efficient. This has been recognized early on $[11,12]$ and it is used in a practically all of the above-cited applications. The perfection of interface engineering with pulsed bias led to the development of Metal Plasma Immersion Ion Implantation and Deposition (MePIIID) [11-15], a combined ion implantation and deposition process.

It is often desirable to process a substrate from all sides in a more or less uniform manner, and this requires the presence of uniform and isotropic plasma around the substrate. In the plasma immersion process using gaseous plasma, also known as plasma source ion implantation (PSII) [16-19], emphasis is put on uniform plasma generation. To check for uniform processing one could measure the sheath thickness and evolution on the different sides of the substrate. Various theoretical models and simulation [20-28] were developed to explain the sheath behavior. Some authors calculated the sheaths around a substrate with complex shapes [29-32]. At the same time, experimental results on sheath evolution were also reported [33-36]. These contributions provide a physical framework for process applications. However, the models generally do not account for supersonic ion velocities and the condensation (sticking and/or subplantation [37]) of plasma ions on surfaces, i.e. effects that are typical for the flowing cathodic arc metal plasmas [38, 39]. Clearly, the plasma densities upstream and downstream of the substrate must be very different, and one can expect large variations in the sheath properties [40].

Since the ion velocity in cathodic arc plasmas $\left(\sim 10^{4} \mathrm{~m} / \mathrm{s}\right)$ is supersonic with respect to the ion sound velocity [38], a cone-shaped volume void of plasma downstream of the flow-impeding object could expected, which is known as the wake effect. The wake effect has been studied since the 1950s to understand the plasma environment of satellites in the upper ionosphere [41]. Recently, wake effects have also been found at the very small scale, for example when a flow of fast ions interacts with nanotubes [42]. In this contribution we avoid the difficulties associated with the very large or very small scales and choose a convenient laboratory plasma, the cathodic arc plasma, and introduce a long-probe technique of measurement. In the case of our flowing arc plasma, the wake is not expected to be completely empty due to the "earlier" plasma which interacted with walls and other components to caused turbulent mixing leading to a low density zone in the back of the substrate - we consider this zone of very low density the wake for the case of a supersonic laboratory arc plasma. When the substrate is pulsed biased to a high negative potential, this wake zone will be occupied by an extended high voltage sheath.

Most of the reported sheath measurements are based on moving a small probe to different positions, or using several probes positioned at various distances from the biased substrate as to observe the passage of the sheath edge [40]. The probes are usually positively biased to collect plasma electrons. If the sheath expands and the probe is not in the plasma but inside the sheath, practically no electrons can be collected. In that sense, the (small) 
probe is a simple yet powerful indicator for the presence or absence of a sheath, however, it is not convenient - or even possible - to continuously record the position of the sheath edge in a single pulse. To observe and measure the whole variation (expansion and recovery) of the sheath during one high voltage pulse (HVP), a long probe could be used. Its length should be greater than the estimated sheath size. Such attempt is made in this contribution. The technique is applied to the case of fast flowing arc plasma where the downstream sheath width is expected to be much greater than the upstream sheath width. The long probe is used in downstream and side position relative to the spherical substrate.

\section{Measurement principle}

The principle of the experiment is shown in Fig. 1. The sheath around a negatively biased substrate is investigated by positively biased, relatively long probes. The terms "negatively biased" and "positively biased" are in reference to the plasma potential, however, for practicality reasons, the ground (chamber, anode) potential is the actual reference - the error introduced is insignificant since the applied bias potentials are large compared to the difference between plasma potential and ground potential.

A negative high voltage pulse (HVP) is applied to the substrate to produce a dynamic sheath. Depending on the applied voltage and local plasma properties, the sheath will expand until a new equilibrium position has been established, as described by many of the models cited in the introduction. For times exceeding the inverse ion plasma frequency,

$$
t>\omega_{p l, i}^{-1}=\left(\frac{\varepsilon_{0} m_{i}}{n_{i} \bar{Q}^{2} e^{2}}\right)^{1 / 2},
$$

the equilibrium thickness is well approximated by the Child-Langmuir law

$$
S_{\text {Child }}(t)=\frac{\sqrt{2}}{3} \lambda_{D e}(t)\left(\frac{2 e V(t)}{k_{B} T_{e}}\right)^{3 / 4},
$$

where the sheath voltage $V(t)$ may be a slowly changing variable;

$$
\lambda_{D e}(t)=\left(\frac{\varepsilon_{0} k_{B} T_{e}}{n_{e}(t) e^{2}}\right)^{1 / 2} .
$$

is the Debye length, $T_{e}$ and $n_{e}$ are the electron temperature and density at the sheath edge,

respectively, $m_{i}, n_{i}$, and $\bar{Q}$ are the ion mass, density and mean charge state number; $k_{\mathrm{B}}$ is the Boltzmann constant, $e$ is the elementary charge, and $\varepsilon_{0}$ is the permittivity of free space.

For a given positive probe potential $U_{p}$ a thin sheath forms around the probe, and the probe collects the electron current $I_{0}$. If we assume, for the moment, that the plasma density is uniform along the probe length $L_{0}$, then the electron current $I_{0}$ should be approximately proportional to the length of the probe $L_{0}$,

$$
I_{0}=k L_{0},
$$


where $k$ is a factor representing the current collected by per unit length. Some part of the probe may be inside the sheath, and another part may be still exposed to the plasma. The probe current will decrease proportionally to the increase of probe section that is inside the sheath. The use of a constant factor $k$ implies that the plasma density can be considered approximately constant outside the sheath, which is of course a simplifying assumption but justified by the huge difference of electron density inside and outside the sheath.

When the applied HVP is terminated, the sheath retreats and the probe current will return to the original value $I_{0}$, provide the plasma generation is still ongoing at the same level.

Possible refinements by considering the presheath are likely to be insignificant because the electron density difference between inside the sheath and the plasma region is very large. We should also recall that the arc plasma under consideration tends to fluctuate greatly due to the noisy plasma generation [43], and therefore those fluctuations would mask any subtle effects of the presheath density. We should also note that the presheath in stationary conditions has a density smaller the density of the undisturbed plasma. In the case of the HVP, however, the electron density can briefly be enhanced because the electrons of the pre-pulse presheath are pushed away by the expanding sheath.

The reduction of the probe current $\Delta I_{\mathrm{p}}(t)$ will be proportional to the length of probe exposed to the sheath $\Delta L(t)$,

$$
\Delta I_{p}(t)=k \Delta L(t)
$$

Combining (4) and (5) gives

$$
\Delta L(t)=\frac{\Delta I_{p}(t)}{I_{0}} L_{0} .
$$

By including the gap $d_{0}$ between the probe tip and the surface of substrate, the actual thickness of sheath can be expressed as

$$
S(t)=d_{0}+\Delta L(t)
$$

with $\Delta L(t)$ of equation (6). It is clear that one will see different regimes of the change of current depending on the relation of probe length and sheath thickness, namely

$$
\Delta I_{p}(t)=\left\{\begin{array}{cll}
0 & \text { for } & S(t)<d_{0} \\
k \Delta L(t) & \text { for } & d_{0}<S(t)<L_{0} \\
I_{0} & \text { for } & S(t)>L_{0}
\end{array}\right.
$$

Based on these simplified considerations, we can obtain information on the sheath expansion and retreat by simply monitoring the variation of one probe current.

\section{Experimental setup}

The experimental setup is shown in Fig. 2. Titanium (Ti) plasma is generated by a pulsed cathodic arc plasma source of the "minigun" type [44] and transported through an open-coil $90^{\circ}$ magnetic filter which is connected in series with the anode of the arc source [45]. The arc source was fed by a pulse forming network [46] delivering a pulsed current of 
$800 \mathrm{~A}, 1 \mathrm{~ms}$ duration, 1 pulse per second. The substrate was a stainless steel sphere with one inch $(25.4 \mathrm{~mm})$ diameter placed $30 \mathrm{~cm}$ from the exit of the magnetic filter.

The bias to the substrate was supplied by a custom tube-switched $20 \mathrm{kV}, 10 \mathrm{~A}$ pulse modulator (NorthStar Research Corp.). The HVPs applied to the substrate included the various combinations of 2,5 , or $10 \mathrm{kV}$ amplitude with a width of 2,4 , or $10 \mu \mathrm{s}$.

Two cylindrical probes with diameter $0.7 \mathrm{~mm}$ were used. One of them, with a length of $25 \mathrm{~mm}$, was position on the side of the sphere, with its axis perpendicular to the plasma flow direction. The other had a length of $50 \mathrm{~mm}$ and was placed downstream, i.e. in the "shadow" or wake of the sphere (not shown in Fig. 2). The distance between the tip of the probes and the sphere surface was $2.5 \mathrm{~mm}$. The probe axis was always normal to the surface of the biased sphere, which is along the direction of sheath propagation.

The probe potential $U_{p}$ was provided by a DC power supply stabilized via capacitors $\mathrm{C}_{1}$ and $\mathrm{C}_{2}$. This was necessary because the fast changing, high current of electrons to the probe would cause an uncontrolled change of potential without those capacitors. The arc, probe, and substrate currents were monitored by broadband Pearson current monitors. The HVP applied to the substrate was monitored by a $20 \mathrm{MHz}, 1000: 1$ high voltage probe, which was directly connected to the substrate. All of signals were recorded by a four-channel digital oscilloscope (Tektronix TDS3014B).

The background pressure of the cryogenically pumped vacuum chamber was about $10^{-4} \mathrm{~Pa}$, and all experiments were done without adding any process gas.

\section{Results and discussion}

\subsection{Probe current waveforms}

Sheath expansion and retreat are clearly visible from the probe signal in Fig. 3 for a typical example of a vacuum arc plasma pulse. By measuring the maximum amplitude of the probe current reduction, $\max \left(\Delta I_{p}\right)$, and using equation (7), the maximum sheath size $S_{\text {max }}$ can be obtained. The dependence of the results on amplitude and duration of the HVP is shown in Fig. 4.

\subsection{Thickness of the sheath}

Figure 4 shows that the sheath signals (i.e. variations of the probe current) are increasing with the amplitude of HVPs and that the retreat of the sheath follows the decay characteristic of bias, i.e., at any given moment in the retreat phase, the sheath thickness adjusts to the voltage. The maximum sheath size produced by different HVPs is shown in Fig. 5. One can clearly see that the sheath downstream is much thicker than the sheath on the side of the sphere. This is expected because the density on the side of the blocking sphere is much greater than the density downstream.

A quantitative analysis of the sheath shows that the thickness does not exactly show the $s \propto V^{3 / 4}$ relationship one might expect from equation (2). This can be explained by re-writing Equ.(2) using the density and temperature dependencies of the Debye length (3), 
leading to

$$
s_{\text {Child }}(t) \propto \frac{V^{3 / 4}(t)}{n_{e}^{1 / 2}(t) T_{e}^{1 / 4}(t)}
$$

where $n_{e}$ and $T_{e}$ are the electron density and temperature at the sheath edge, respectively.

As the negative HVP is applied to the sphere, electrons are pushed away to make space for the thicker sheath. Those accelerated, energetic electrons interact with the plasma electrons, and it is clear that - temporarily - the density and temperature of the electrons in the region outside sheath are enhanced. Therefore, according to (9), the sheath thickness should not expand as much as $V^{3 / 4}$.

We can further notice that the sheath expansion on the side of the sphere directly affects the sheath downstream at a later time, which will be further discussed below.

The relatively long decay time of the applied HVP allowed the sheath to follow correspondingly. To further explore the features of a retreating sheath, the fall time of the HVP should be reduced. A shorter HVP fall time was achieved by installing an additional ohmic load resistor between the biased sphere and ground. This resistor, sometimes dubbed "tail biter" ([47]), is indeed very effective, compare Figs. 6 and 7.

The following results refer to sheath properties when using the improved HVP of shorter fall time. The sheath's steady state duration depends on the width of the HVP, indicating the sheath has reached the equilibrium position associated with the local plasma properties. The expansion and retreat of the sheath on the side (Fig. 7) are faster than the corresponding times downstream (Fig. 6). There was almost no delay between the response of the sheath on the side and the application of the HVP. In contrast, the sheath downstream has a noticeable longer propagation time, and in fact the sheath did not stop expanding even after the HVP was switched off, see for example the case of $2 \mu \mathrm{s} \mathrm{HVP} \mathrm{(Fig.} \mathrm{6,} \mathrm{see} \mathrm{also} \mathrm{Fig.} \mathrm{4).}$ Without flow, the sheath retreat would have followed a line BC (Fig. 6). Expulsing the plasma on the side by applying a HVP caused a reduced plasma density downstream at a later time, i.e. when the HVP is over. Therefore, the downstream sheath expands from B to D and finally retreats from $\mathrm{D}$ to $\mathrm{E}$ (Fig. 6).

\section{Summary and conclusions}

In this work we have demonstrated that information on the sheath expansion and collapse caused by a high voltage pulse can be observed and recorded by using a single long probe. To measure sheath features at different positions, two or more probes can be used. In the present experiment, the substrate was a sphere immersed in flowing titanium plasma, and the sheath on the side and downstream was monitored for various high voltage pulses up to $10 \mathrm{kV}$ and with a duration as short as $2 \mu \mathrm{s}$. Taking the sheath models developed for plasma immersion ion implantation, the equilibrium sheath is a self-adjusting Child-Langmuir sheath, which depends on both applied voltage and plasma parameters. As expected, the sheath size downstream is much larger than on the side of the biased sphere. The technique of a long probe nicely illustrates that sheath expansion downstream of the substrate is delayed 
with respect to the bias pulse and because the downstream density is affected by the expulsion of plasma on the side.

\section{Acknowledgments}

The work was supported in part by the China Scholarship Committee and in part by the U.S. Department of Energy under Contract No. DE-AC02-05CH11231. 


\section{References}

[1] Anders A, Fong W, Kulkarni A, Ryan F R, and Bhatia C S IEEE Trans. Plasma Sci. 200129 768-775.

[2] Robertson J Thin Solid Films 2001383 81-88.

[3] Casiraghi C, Ferrari A C, Ohr R, Chu D, and Robertson J Diamond Rel. Mat. 200413 1416-1421.

[4] Siemroth P, Wenzel C, Kliomes W, Schultrich B, and Schülke T Thin Solid Films 1997 308 455-459.

[5] Monteiro O R J. Vac. Sci. Technol. B 199917 1094-1097.

[6] Siemroth P and Schülke T Surf. Coat. Technol. 2000 133-134 106-113.

[7] Chang Y-Y, Yang S-J, and Wang D-Y Thin Solid Films 2007515 4722-4726.

[8] Hörling A, Hultman L, Odén M, Sjölén J, and Karlsson L Surf. Coat. Technol. 2005 191 384-392.

[9] Tay B K and Zhang P Thin Solid Films 2002 420-421 177-184.

[10] Anders A J. Phys. D: Appl. Phys. 200740 2272-2284.

[11] Brown I G, Godechot X, and Yu K M Appl. Phys. Lett. 199158 1392-1394.

[12] Brown I G, Anders A, Anders S, Dickinson M R, Ivanov I C, MacGill R A, Yao X Y, and Yu K-M Nucl. Instrum. Meth. Phys. Res. B 1993 80/81 1281-1287.

[13] Anders A, Anders S, Brown I G, Dickinson M R, and MacGill R A J. Vac. Sci. Technol. B 199412 815-820.

[14] Anders A Surf. Coat. Technol. 199793 157-167.

[15] Bilek M M M, McKenzie D R, Tarrant R N, Lim S H N, and McCulloch D G Surf. Coat. Technol. 2001156 136-142.

[16] Conrad J R, Radtke J L, Dodd R A, Worzala F J, and Tran N C J. Appl. Phys. 198762 4591-4596.

[17] Xia Z and Chan C J. Appl. Phys. 199373 3651-3656.

[18] Wood B P J. Appl. Phys. 199373 4770-4778.

[19] Anders A (Ed.) Handbook of Plasma Immersion Ion Implantation and Deposition. New York: John Wiley \& Sons, 2000.

[20] Lieberman M A J. Appl. Phys. 198966 2926-2929.

[21] Günzel R and Brutscher J Surf. Coat. Technol. 1996 85 98-104.

[22] Hong M and Emmert G A J. Vac. Sci. Technol. B 199412 889-896.

[23] Riemann K U and Daube T J. Appl. Phys. 199986 1202-1207.

[24] Sheridan T E and Alport M J Appl. Phys. Lett. 199464 1783-1785.

[25] Wang D, Ma T, and Deng X J. Appl. Phys. 199475 1335-1339.

[26] Dai Z L and Wang Y N J. Appl. Phys. 200292 6428-6433.

[27] Miyagawa Y, Ikeyama M, Miyagawa S, and Nakadate H Nucl. Instrum. Meth. Phys. Res. B 2003206 767-771.

[28] Sheridan T E and Goeckner M J J. Appl. Phys. 199577 4967-4972.

[29] Sheridan T E J. Phys. D: Appl. Phys. 199528 1094-1098.

[30] Donnelly I J and Watterson P A J. Phys. D: Appl. Phys. 198922 90-93.

[31] Watterson P A J. Phys. D: Appl. Phys. 198922 1300-1307.

[32] Sheridan T E IEEE Trans. Plasma Sci. 199624 57-58.

[33] Shamim M, Scheuer J T, and Conrad J R J. Appl. Phys. 199169 2904-2908. 
[34] Brutscher J, Günzel R, and Möller W Plasma Sources Sci. Technol. 19965 54-60.

[35] Kim Y-W, Kim G-H, Han S, Lee Y, Cho J, and Rhee S-Y Surf. Coat. Technol. 2001 136 97-101.

[36] Yatsuzuka M, Miki S, Morita R, Azuma K, and Fujiwara E Surf. Coat. Technol. 2001 136 93-96.

[37] Lifshitz Y, Kasai S R, Rabalais J W, and Eckstein W Phys. Rev. B 199041 10468-10480.

[38] Anders A and Yushkov G Y J. Appl. Phys. 200291 4824-4832.

[39] Byon E and Anders A J. Appl. Phys. 200393 1899-1906.

[40] Anders A Surf. Coat. Technol. 2001136 85-92.

[41] Engwall E, "Numerical studies of spacecraft-plasma interaction: simulations of wake effects on the cluster electric field instrument EFW (IRF Scientific Report 284)," Swedish Institute of Space Physics, Uppsala, Sweden, 2004.

[42] Mowbray D J, Miskovic Z L, Goodman F O, and Wang Y-N Phys. Lett. A 2004329 94-99.

[43] Anders A IEEE Trans. Plasma Sci. 200533 1456-1464.

[44] MacGill R A, Dickinson M R, Anders A, Monteiro O R, and Brown I G Rev. Sci. Instrum. 199869 801-803.

[45] Anders A IEEE Trans. Plasma Sci. 200230 108-109.

[46] Anders A, MacGill R A, and McVeigh T A Rev. Sci. Instrum. 199970 4532-4534.

[47] Goebel D M, Adler R J, Beals D F, and Reass W A, "Pulser Technology," in Handbook of Plasma Immersion Ion Implantation and Deposition, Anders A, Ed. New York: Wiley, 2000, 467-513. 


\section{Figure Captions}

Fig. 1 Illustration of the sheath size measurements: The substrate is a sphere, immersed in plasma and surrounded by a large sheath when biased at a high negative voltage. The probe is a positively biased wire that may be partially inside the sheath and partially exposed to plasma. The asymmetry in the sheath is caused by the supersonic plasma flow.

Fig. 2. Setup and electrical schematic of the experiment using long probes for the measurement of the sheath dynamics. Flowing metal plasma is generated by a pulsed filtered cathodic arc, streaming toward to biased sphere. For clarity, only the probe on the side is shown; the actual experiment used a second probe downstream.

Fig. 3. Typical data set, here for the downstream probe: the pulse waveforms show arc current, probe current, and substrate current; the applied HVP was $2 \mathrm{kV}$ with a nominal duration of $4 \mu \mathrm{s}$.

Fig. 4. Time dependent current to the downstream probe for various HVP: (a): $2 \mathrm{kV}$, (b): 5 $\mathrm{kV},(\mathrm{c}): 10 \mathrm{kV}$.

Fig. 5 Maximum sheath size determined by the downstream and side probes for the various bias pulse duration and amplitudes, as indicated.

Fig. 6 Current to the downstream probe, indicative for the downstream sheath, with the modified HVP having a faster fall time. Note that the response of the downstream sheath to the end of the pulse is delayed, indicative of the flow effect on the downstream sheath.

Fig. 7 As figure 6, but measured on the side of the biased sphere: here, the sheath response the end of the HVP is not delayed. 


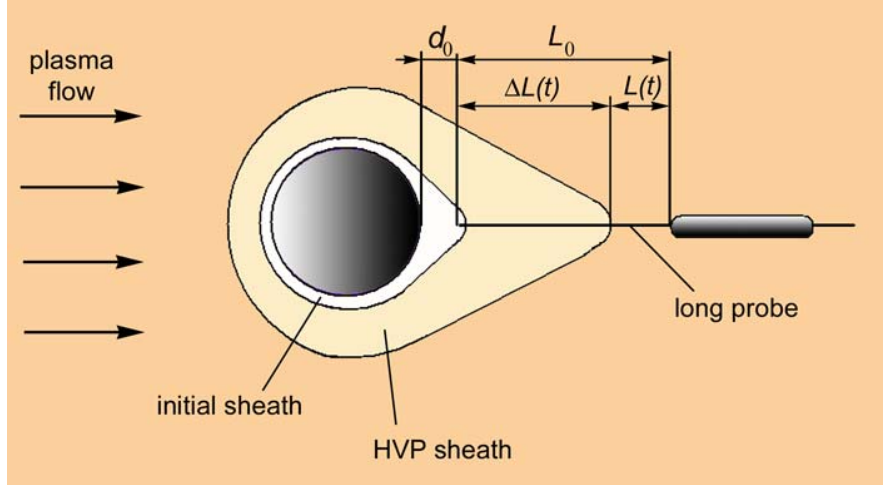

Fig. 1 


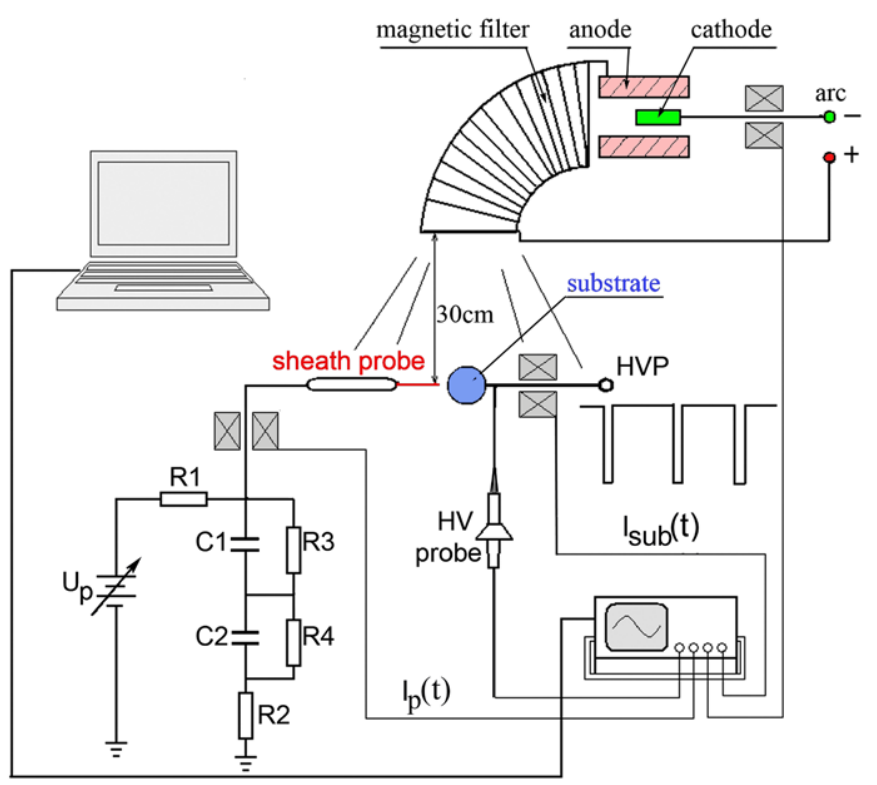

Fig. 2 


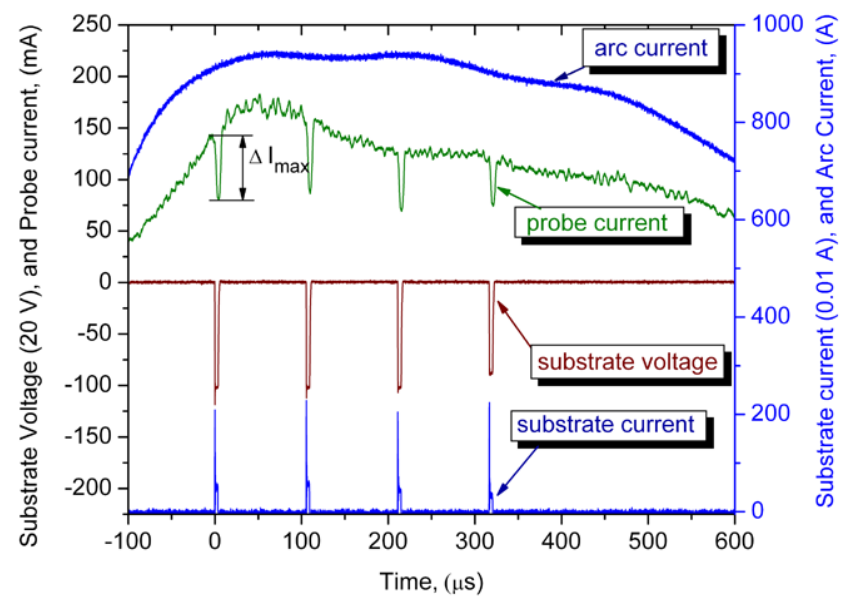

Fig. 3 

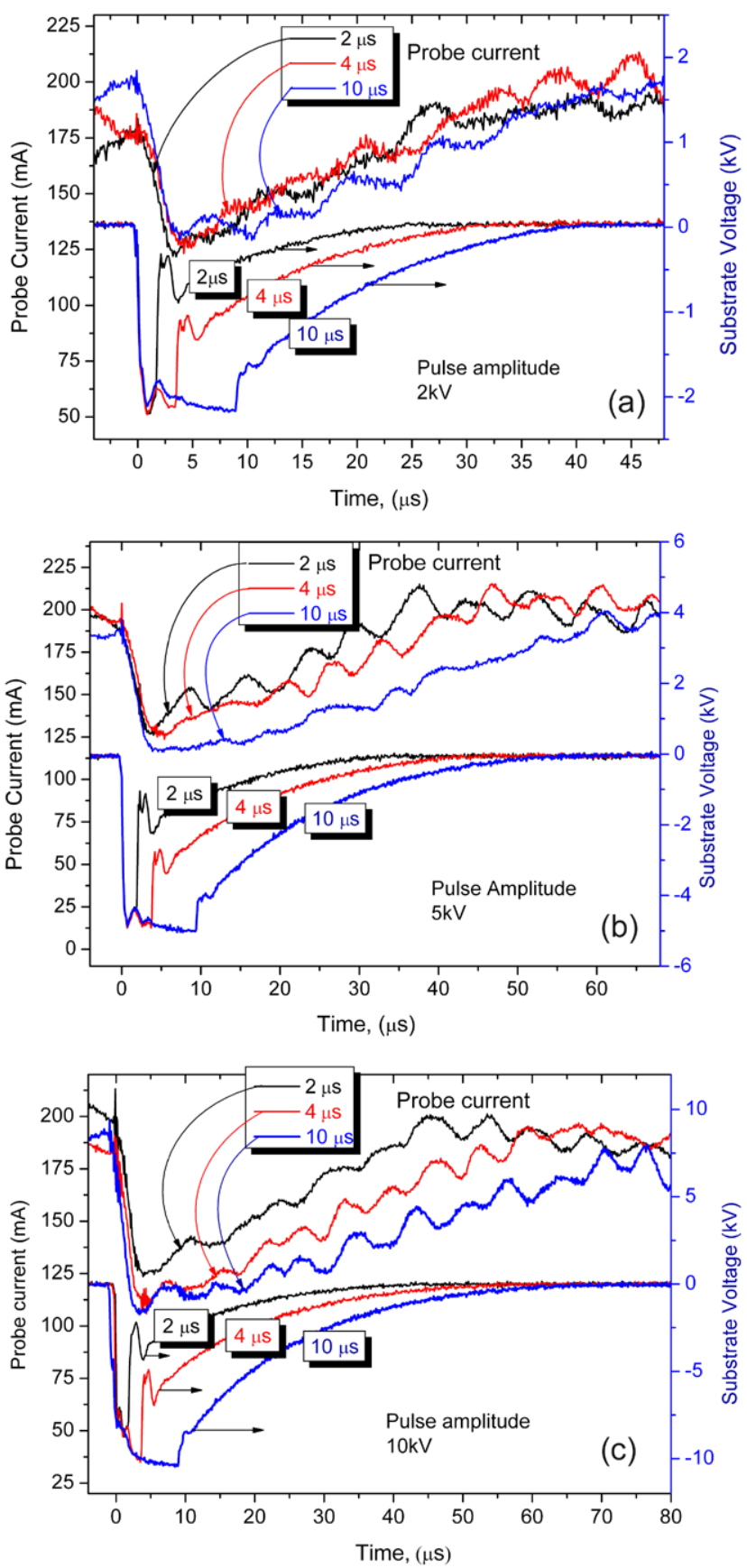

Fig. 4 


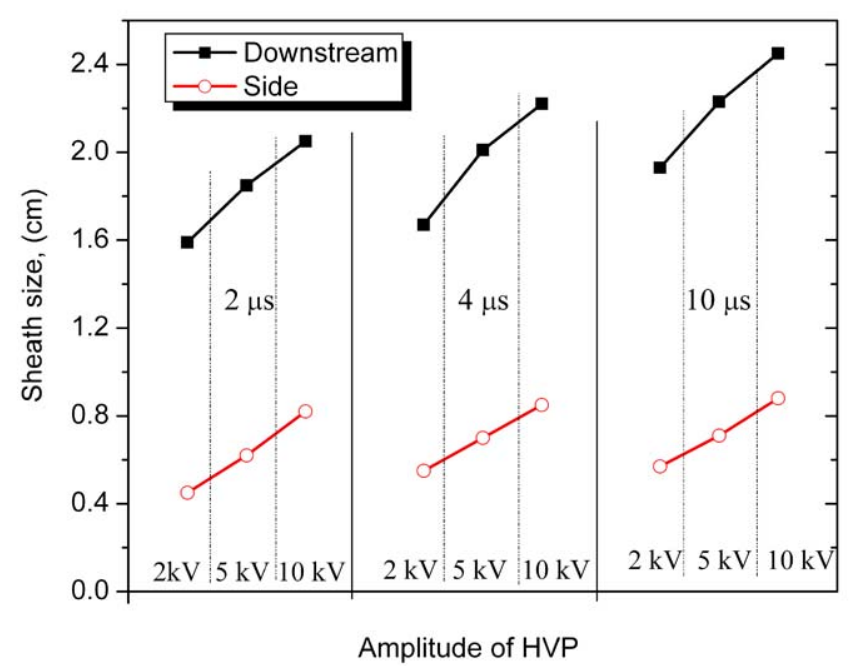

Fig. 5 


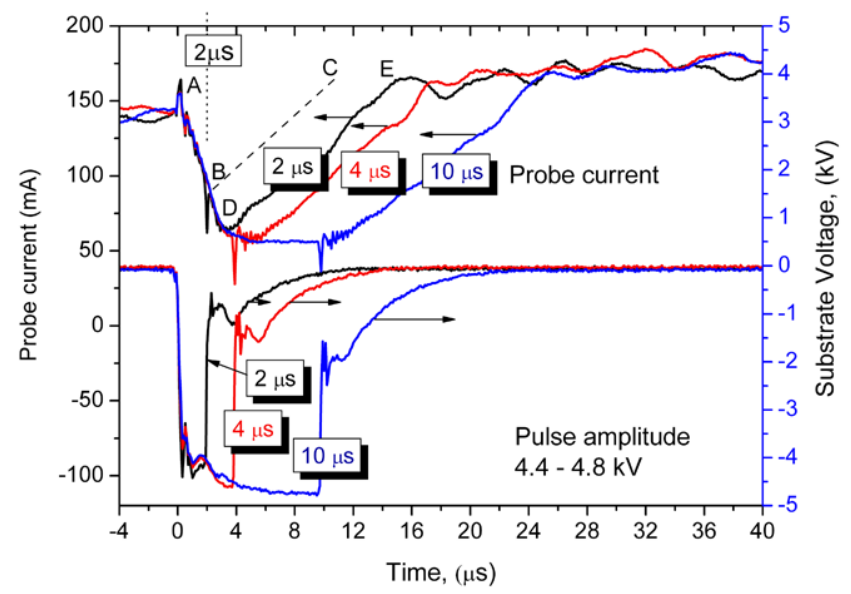

Fig. 6 


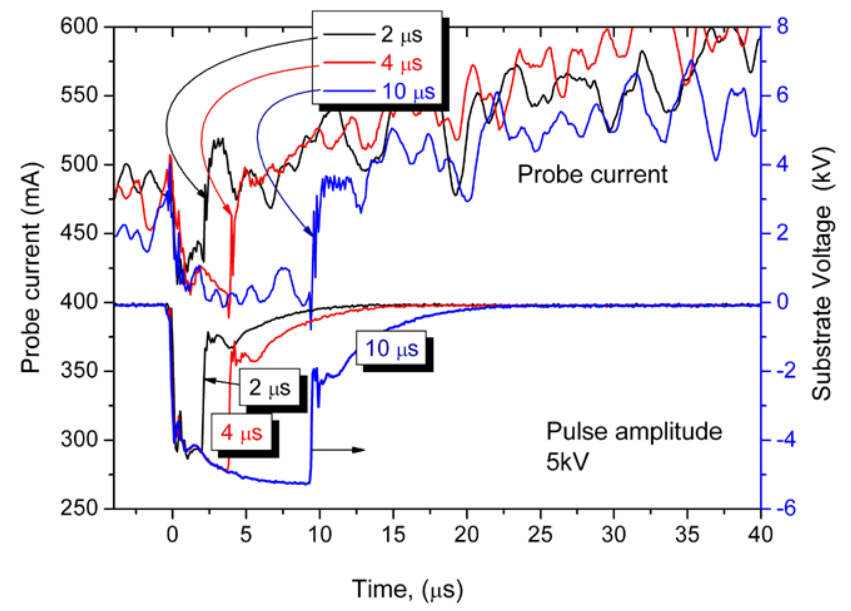

Fig. 7 